\title{
Analysis of velocity and acceleration trends using slope stability radar to identify failure 'signatures' to better inform deformation trigger action response plans
}

\author{
R Shellam SRK Consulting (UK) Limited, UK \\ J Coggan University of Exeter, UK
}

\begin{abstract}
Ground strata failure is a major hazard for open pit mines as it has the potential to cause damage to property and can result in multiple fatalities. Slope stability radar (SSR) systems are used to continuously monitor pit walls and they can detect slope deformation to sub-millimetre scales. However, geotechnical engineers may have limited prior data to set the required trigger action response plan (TARP) thresholds. As a result, arbitrary values or data from other sites are often used to signify dangerous deformation rates which can ineffectively trigger alarms.
\end{abstract}

Therefore, the primary aim of this investigation was to identify the failure indicator factors which best inform TARP thresholds at a particular mine site. Data from eight open pit failures from the same mine were analysed and then compared with data from other published failures. A secondary aim was to a develop a database of combined failure events that could be used as a reference to set meaningful TARP threshold levels at other mine sites with similar mining conditions.

The study site failure events ranged in size from 200 to 200,000 tonnes, with most failures occurring in the upper part of the slopes within highly to completely weathered rock. Geological and geotechnical characteristics of the rock mass for the observed failure modes were also included in the analysis. Multiple peak acceleration and peak velocity plots were used to determine clustering for the different characteristics investigated. It was shown that as the failure size increased so did the peak velocity, suggesting that larger failures can accommodate higher displacement rates. Analysis of the combined dataset showed a clear positive relationship between failure size and failure mode up to approximately 3,000 t. However, failure events greater than 3,000 t do not appear to have clear grouping by failure size, suggesting that other factors may control the peak acceleration and velocity rates. This suggests that the TARP should consider different trigger thresholds based on the expected failure mode and size. However, the accurate recording of all failure data across sites with additional characteristics such as, Rock Mass Rating (RMR ${ }_{89}$ ), GSI, weathering and lithology would enable improved analysis of velocity trends to provide further insights into factors influencing potential failure.

It is concluded, that back-analysis of slope instability events using log-acceleration and log-velocity plots can refine thresholds used in TARPs for specific sites with the study site TARP presented. However, the consistent collection, processing and filtering of failure data across sites is required to improve analysis and implementation of findings.

Keywords: slope stability radar (SSR), trigger action response plan (TARP), open pit, slope failure

\section{Introduction}

Many open pit mines implement slope stability radar (SSR) monitoring systems as part of the hazard management plan to mitigate the risk associated with slope failure. Technology has advanced with systems able to collect data both automatically and from multiple sources (e.g. SSR and Interferometric Synthetic Aperture Radar (InSAR)). This has vastly improved the quality and quantity of information available for 
monitoring and subsequent analysis. However, a robust geotechnical monitoring program is still required to confirm the data is being effectively analysed and communicated for safe day-to-day mining operations (Read \& Stacey 2009; Osasan \& Afeni 2010; Bar et al. 2016).

The aim of the project was to identify failure signatures which could help inform trigger action response plan (TARP) thresholds for a particular mine site and assess whether this could be applied to other mine sites with similar geotechnical and mining conditions. To achieve this, eight open pit instability events monitored by SSR were analysed. In addition to the SSR data, geological and geotechnical descriptions of the rock mass characteristics were included within the database to identify smaller subsets that may be present within the case examples investigated. Finally, the results from this study were compared with published results to provide a larger dataset for interpretation.

\subsection{Trigger action response plans}

The TARP is a workflow procedure that comprises of trigger thresholds for different sensors based on the system measurement type or geographical location. Typically, geotechnical slope TARPs have 'green', 'orange' and 'red' trigger levels which may relate to changes in deformation (velocity and/or acceleration), visual observations, rainfall or water levels (porewater pressure) indicating increased likelihood of slope failure and the associated risk. The TARP is a critical part of the slope monitoring program, as it clearly details when monitoring results should be deemed dangerous and appropriate actions taken for remediation (Maton 2002; Read \& Stacey 2009; Bar et al. 2016). This paper focuses specifically on the slope deformation TARP.

It is widely accepted that a TARP needs to be tailored to the conditions at a specific mine site following a detailed risk assessment which includes modes of instability and slope instability risk (Read \& Stacey 2009). The most effective method for calibrating a TARP is to analyse monitoring results from past failure events (Carla et al. 2017a). Therefore, developing an effective TARP can be difficult for onsite geotechnical engineers when there is an absence of historical data. As a result, arbitrary values or data from other sites are used to define threshold deformation rates which can ineffectively trigger alarms as not all exceedances of threshold values are dangerous. A key objective of this project is to present several past slope failures monitored by SSR units that can provide reference values and help select rational trigger levels for mines sites with similar ground conditions.

\subsection{Previous work}

The analysis of slope failures developed from fundamental geotechnical concepts which were identified from small-scale laboratory testing in the 1950s (Saito 1965; Fukuzono 1985). The evolution of modern monitoring systems (e.g. SSR), which can detect surface displacement at a sub-millimetre scale with a high sampling rate, has validated many of these concepts. However, this has also provided a vast scope for additional research into failure mechanisms and associated deformation.

Initially, research associated with radar systems was focused on refining time of failure (TOF) prediction methods for specific slope failures (Rose \& Hungr 2007; Harries et al. 2009; Arosio \& Harries 2010; Venter et al. 2013; Dick et al. 2015). Two eminent methods of analysis were used: inverse velocity and slope gradient (SLO). Subsequently, the research focus shifted to identifying 'signature' parameters across multiple events with the aim of providing TARP thresholds (Carla et al. 2017a; Crosta et al. 2017; Farina et al. 2018b).

\subsubsection{Failure prediction methods}

Mufundirwa et al. (2010) developed the SLO method to predict the 'life expectancy' of failure events by plotting the displacement velocity, multiplied by time on the $y$-axis and the displacement velocity on the $x$-axis. TOF is obtained by calculating the slope of the resulting graph (Mufundirwa et al. 2010; Venter et al. 2013). The SLO method was used by Venter et al. (2013) on a number of failure examples, showing that the results were sensitive to data averaging for the velocity calculation, in addition to the data period used in the slope calculation. Also, Venter et al. (2013) noted that the abstract nature of the SLO graphs was difficult for 
practitioners to easily use, particularly during a failure event. As a result, the SLO method has not been widely adopted by the mining industry.

Rose \& Hungr (2007) comprehensively applied the inverse velocity method of Fukuzono (1985) to four open pit failures using data collected from robotic total station. They concluded that the method was a powerful tool which significantly improved the ability to interpret monitoring data and estimate the TOF.

Dick et al. (2015) effectively applied SSR data from eight slope failures to predict TOF using the inverse velocity (Fukuzono 1985) and slope gradient (Mufundirwa et al. 2010) methods. Dick et al. (2015) proposed new techniques for SSR data filtering, as opposed to traditional data (e.g. prisms) due to the high spatial and sampling rate of SSR data. A single and multi-pixel selection procedure, named the 'percent deformation method' was shown to improve the TOF predictions. In addition, Dick et al. (2015) highlighted the necessity to incorporate these systematic data filtering and analysing procedures into the TARP to ensure TOF predictions were consistent.

Carla et al. (2017a) presented nine slope instability events in a brittle hard rock mine. The brittle rock mass resulted in rapid accelerations just prior to failure. Therefore, the short tertiary creep phase was making failure prediction challenging. Carla et al. (2017a) highlighted that the onset of slope creep stages (primary, secondary, tertiary) were significantly different depending on the failure size, rock type and/or failure mode.

There are many other successful examples of TOF predictions implemented at study sites providing validity to the methods presented above (e.g. Harries et al. 2006, 2009; Arosio \& Harries 2010; Carla et al. 2017a). The inverse velocity technique is the most widely used method in the mining industry due to the relatively simple analysis and interpretation of data (Cabrejo-Lievano 2013). However, it is widely accepted that an early warning failure prediction is only effective if calibrated and contextualised to the onsite slope characteristics and deformation behaviour. As a result, recent research has been focused towards identifying failure 'signatures', some of which are outlined further.

\subsubsection{Failure signature identification}

Identifying failure signatures was initially proposed by Federico et al. (2012) when analysing the relationship between peak velocity and peak acceleration at the point of failure for 38 events. Federico et al. (2012) proposed a linear relationship between the log-acceleration and log-velocity just prior to failure. They also outlined the practical use of the chart when historical slope displacement data was not available.

Newcomen \& Dick (2016) expanded the velocity-acceleration analysis by including five additional failure examples to the Federico et al. (2012) dataset. Clustering of results by rock type (volcanic versus sedimentary) along the linear trend was observed with volcanic rocks failing at higher velocity and acceleration. Thus, Newcomen and Dick (2016) additionally recommended sorting results by failure mode and rock type to identify smaller subsets and underlying signatures for different failure events.

Carla et al. (2017a) used the velocity-acceleration method to analyse nine open pit slope instability events in hard rock, structurally controlled examples analysing failure and non-failures events. Higher velocity and acceleration rates were observed for failures compared to non-failure events. In addition, smaller failures associated with a single pre-defined discontinuity (i.e. planar) had lower velocity and acceleration rates compared to larger events with multiple discontinuities (i.e. more complex failures mechanisms involving interaction of several discontinuities). Carla et al. (2017a) suggested that large instabilities are likely to consist of numerous interacting blocks moving along a number of different surfaces. This enables the slope to accommodate large velocity and acceleration rates with a greater likelihood of block-interlocking resulting in eventual stability. Whereas, a single rock block moving on a predefined discontinuity (or pair of discontinuities in the case of wedge failures) would only accommodate a small amount of deformation. Therefore, it would be more likely to have lower velocity and acceleration rates. In conclusion, Carla et al. (2017a) recommended all TARP thresholds need to be calibrated to onsite events, where different alarm thresholds are required depending on geometry, size and failure mode properties. 
This research highlights the need for continual updating of databases to learn from previous events and to utilise the recent improvements in data collection and analysis techniques. Therefore, the analysis of new failure events will not only improve the identification of failure signatures, but also aid the improvement of mine safety.

\subsection{Study site location}

The open pit mine study site is located within the African Copperbelt, in the southeastern part of the Democratic Republic of Congo and has been operating since 2006. At the time of writing the site operated three open pits (Central-North West, Central and East Pit). Figure 1 outlines the general layout of the study site indicating the location of the respective open pits, SSR's and slope instability events.

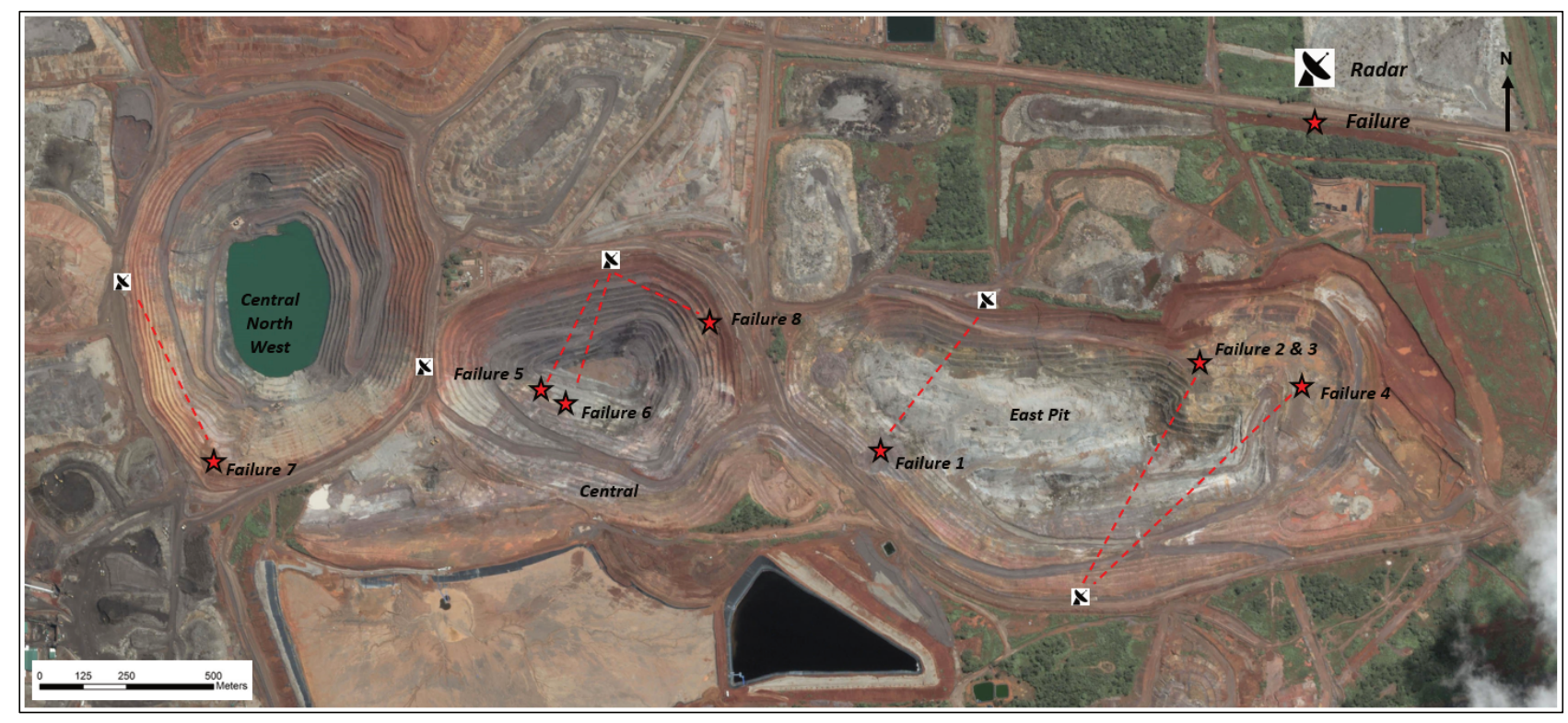

Figure 1 General layout of the open pits at the site with the locations of the slope stability radar (SSR) units and slope instability events

In total, the strike length of the open pits is approximately 3,000 m. All the benches were $10 \mathrm{~m}$ high with varying inter-ramp and overall slope angle based on the combination of geological rock type, weathering classification and structural domain. Typically, inter-ramp angles ranged from $30^{\circ}$ to $45^{\circ}$ depending on the geotechnical sector.

\section{Methodology}

The aim of this investigation was to identify the failure factors which best inform TARP thresholds at the study mine site. Quantitative (SSR deformation data) and qualitative (e.g. geological descriptions failure mode) data from eight open pit failures from the same mine were analysed. A Pearson's correlation matrix was developed using six variables (peak velocity, peak acceleration, failure size, start of tertiary phase, weathering and Rock Mass Rating (RMR)) to identify relationships that may exist across the failure case examples. In addition, multiple peak acceleration and peak velocity plots were used to determine clustering for the different characteristics. This method has been utilised by previous researchers and is one technique for identifying failure signatures (Federico et al. 2012; Newcomen \& Dick 2016; Carla et al. 2017a).

Following this, data from the study site was compared with data from other published failures to develop a larger dataset for analysis using peak acceleration and peak velocity plots. A secondary aim was to a develop a database of combined failure events that could be used as a reference to set meaningful TARP levels at other mine sites with similar mining conditions. 
The impact of SSR sampling frequency, line of sight (LOS) sensitivity and velocity calculation period are critical when deploying SSR units in the field to ensure the system will trigger alarms effectively. Processing steps are required to correct SSR data when completing back-analysis and are described in further detail.

\subsection{Sampling frequency}

The sampling frequency is defined as the rate at which the SSR can scan the target slope. Vaziri et al. (2010) notes that shorter sampling frequency rates aid in the analysis of monitoring data, particularly when attempting failure predictions, thus they suggest scan frequencies of a few minutes. The sampling frequency is important for three reasons:

1. Acceleration rates above the maximum detection rate per scan will cause phase ambiguity and phase wrapping resulting in erroneous results being generated (Crosta et al. 2017). Therefore, shorter scan times will enable a wider range of velocity rates to be detected.

2. Rockfall, wedge and planar failures in hard rock are characterised by a short acceleration phase prior to the collapse (Dick et al. 2015; Carla et al. 2017a). Therefore, if the sampling rate is greater than the acceleration phase, deformation may not be detected.

3. In the late stages of slope instability events, high frequency monitoring data aids failure forecasting by identifying acceleration. In addition, accurate TOF predictions require constant reevaluation as new data becomes available (Rose \& Hungr 2007; Carla et al. 2017b). During critical acceleration events it is recommended to reduce scan time to two minutes (Crosta et al. 2017). Furthermore, with higher sampling frequency more predictions can be performed (Dick et al. 2015).

\subsection{Line of sight sensitivity}

The location of the SSR relative to the slope and failure direction will determine the quantity or magnitude of deformation recorded by the system (Carla et al. 2017a). When the SSR is perpendicular to the slope and direction of failure, $100 \%$ of the total deformation vector will be recorded. However, as the scan angle increases smaller amounts of the total deformation will be detected. Therefore, the LOS is a data processing stage that is required to account for the component of movement not perpendicular to the SSR.

Carla et al. (2017a) recommended kinematic analysis is undertaken to determine the likely direction of failures relative the SSR. Once this is estimated the alarm thresholds can be modified to account for the LOS correction required.

\subsection{Velocity calculation period}

The velocity calculation period (VCP) is the time period used to calculate the rate of deformation (i.e. velocity). Typically, 1, 5 and 24-hour calculation periods are used during a failure event to calculate the velocity. Shorter calculation periods will provide instantaneous velocity rates. However, they may also introduce noise due to instrumental or environmental deformation rates which are similar to the real movement on the wall (Cabrejo-Lievano 2013). Longer calculation periods have a smoothing effect and provide cleaner data for analysing long-term trends. As the velocity increases prior to collapse the averaging effect can provide little forewarning to the event.

Failure \#8 from the study site examples has been used to highlight the effect of the VCP when attempting to predict failures based on Fukuzono (1985) method of linear inverse velocity trends. Figure 2 outlines three $\operatorname{VCP}(1,5$ and 24 -hour) used to predict the TOF 12,4 and 2 hours prior to the collapse (red star indicating the actual failure time). Chart A shows analysis 12-hours prior to collapse while deformation rates were still relatively small. The 24 -hour calculation period provides a coherent failure estimation time due to the longer averaging period. The shorter VCP introduced excessive noise and as a result no discernible trend can be extrapolated to predict failure. However, in Chart C, two hours prior to failure, velocity rates rapidly increased and the one hour calculation period predicts the TOF to within 20 minutes. Chart B, four hours prior to failure, 
vastly under and over predicts the failure time depending on VCP and is therefore of little use to the practitioner. This provides an example of the difficulty encountered when attempting to predict failures at different stages during the event.

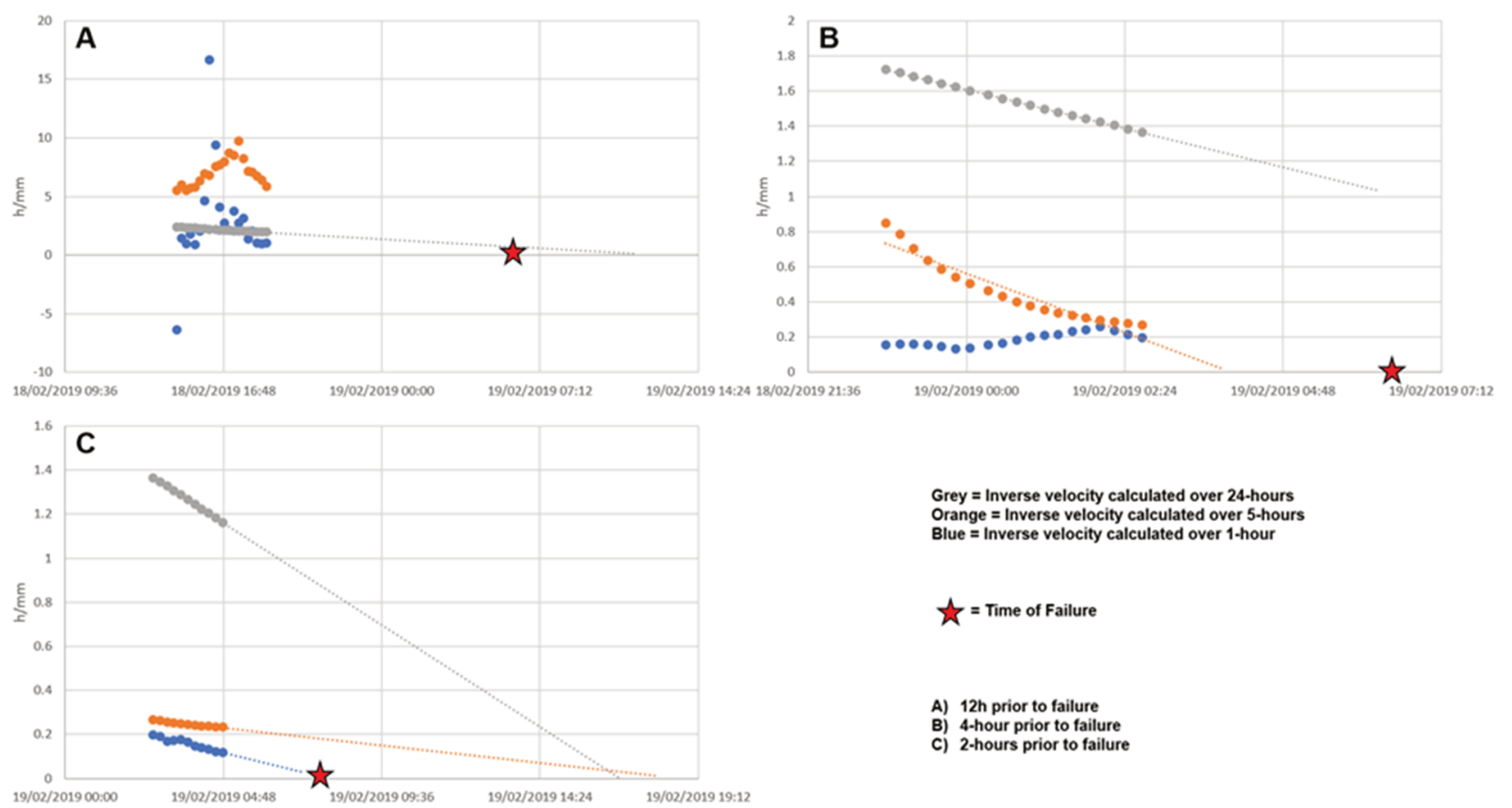

Figure 2 Effect of varying velocity calculation periods to predict time of failure at 12, 4 and 2 hours prior to collapse. Data from Failure \#8 at the study site

No prescriptive method has been developed for determining the VCP due to the variability in failure deformation rates. However, it is a critical data processing step for reliable failure predictions. Cabrejo-Lievano (2013) noted that generally longer VCP's allow early detection of deformation trends. Whereas, shorter VCP's are affected by the most recent deformation state of the rock mass and will provide better TOF predictions close to collapse.

\section{Data}

Eight open pit failures occurring at the study site between 2016 and 2019 form the primary dataset for analysis. These range in size from $200 \mathrm{t}$ to $200,000 \mathrm{t}$ with most of the failures occurring in the upper part of the slopes. Four of the events have been classified as hybrid failures as one limb of the failure developed at a fault or stratigraphical contact introducing a structural control to the event (failure examples \#1, \#2, \#5 \& \#7). Three were rotational failures through weak rock masses (examples \#4, \#6 and \#8) and one event was a secondary slip within a pre-existing failure (example \#3). The results from example \#3 highlight the difficulty when monitoring post-failure deformation trends. However, Failure \#3 results have been excluded in the analysis as the deformation behaviour is related to unconsolidated soil material. Table 1 summaries the failure events. 


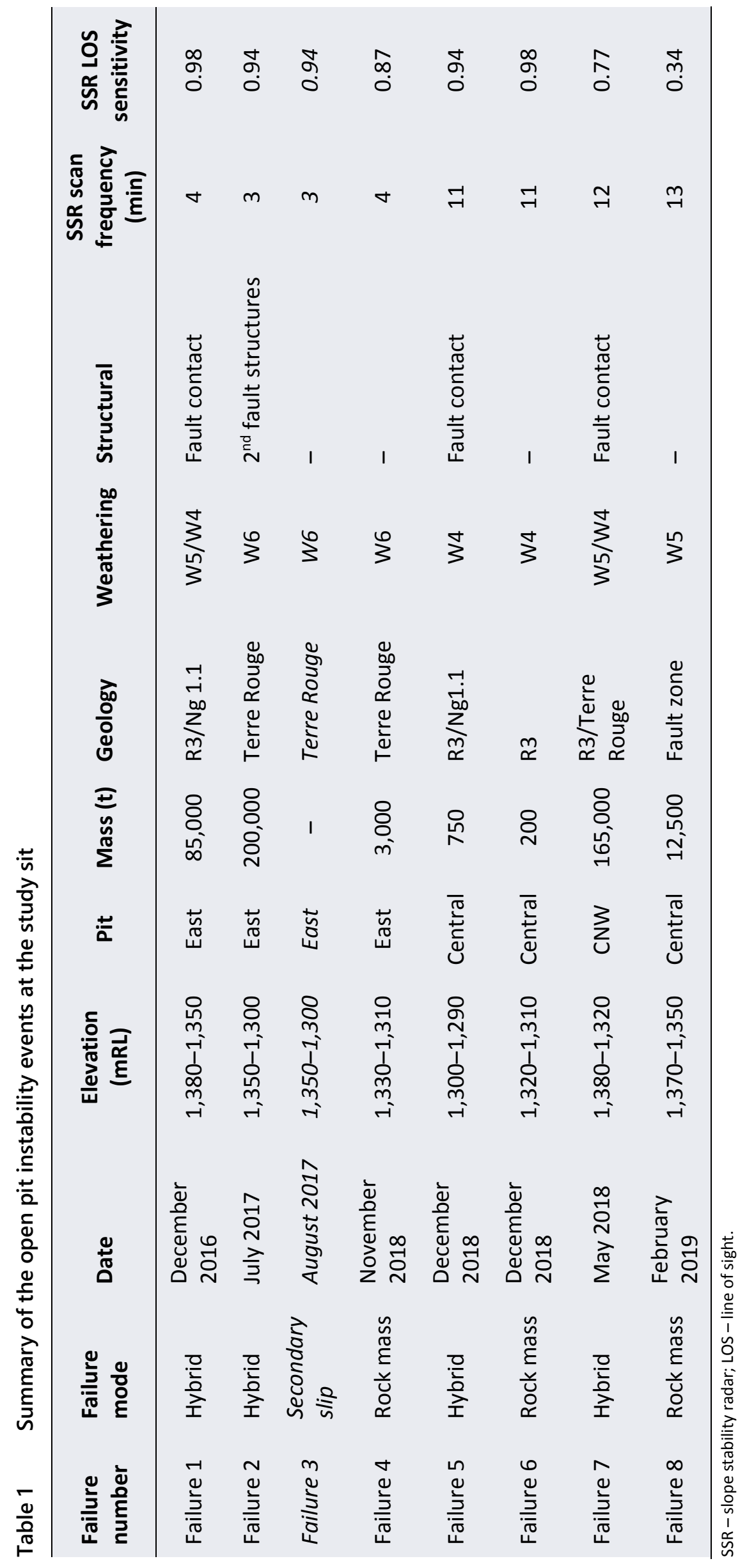


Accompanying the geological and visual description, all the failures were monitored by SSR (Ground Probe SSR-XT) which provides highly accurate and frequent surface displacement measurements. All the measurements recorded by the SSR were corrected for LOS effect (Carla et al. 2017a). A one hour VCP was used in the analysis (Table 2).

Table 2 Corrected line of sight deformation, velocity and acceleration rates for the study site examples

\begin{tabular}{cccc}
\hline $\begin{array}{c}\text { Failure } \\
\text { number }\end{array}$ & $\begin{array}{c}\text { Corrected } \\
\text { deformation }(\mathbf{m m})\end{array}$ & $\begin{array}{c}\text { Corrected peak } \\
\text { velocity }(\mathbf{m m} / \mathbf{h})\end{array}$ & $\begin{array}{c}\text { Corrected peak } \\
\text { acceleration }\left(\mathbf{m m} / \mathbf{h}^{2}\right)\end{array}$ \\
\hline Failure 1 & 270 & 60 & 23.7 \\
Failure 2 & 1,635 & 141.4 & 45.3 \\
Failure 3 & 99 & 26 & 15.3 \\
Failure 4 & 207 & 30.3 & 32.2 \\
Failure 5 & 52 & 7.5 & 6.7 \\
Failure 6 & 205 & 8.7 & 6.7 \\
Failure 7 & 999 & 35.8 & 16 \\
Failure 8 & 114 & 32.7 & 14.4 \\
\hline
\end{tabular}

\section{$4 \quad$ Results}

A Pearson's correlation matrix with $p$ significance values was developed using six variables to identify relationships that may exist across the failure case examples (Table 3). The geological description and failure mode were removed from the dataset as they are nominal variables without a quantitative value.

Table 3 Pearson's correlation matrix with $p$ significant values for six variables for the seven failure events

\begin{tabular}{lcccccc}
\hline & $\begin{array}{l}\text { Peak } \\
\text { acceleration }\end{array}$ & $\begin{array}{l}\text { Peak } \\
\text { velocity }\end{array}$ & $\begin{array}{l}\text { Failure } \\
\text { size }\end{array}$ & $\begin{array}{l}\text { Tertiary } \\
\text { phase }\end{array}$ & Weathering & RMR \\
\hline Peak acceleration $(r)$ & 1 & & & & \\
P-value & - & & & & \\
Peak velocity $(r)$ & $\mathbf{0 . 8 8}$ & 1 & & & & \\
P-value & $\mathbf{0 . 0 1}$ & - & & & \\
Failure size $(r)$ & 0.6 & $\mathbf{0 . 8 1}$ & 1 & & \\
P-value & 0.16 & $\mathbf{0 . 0 3}$ & - & & \\
Tertiary phase $(r)$ & -0.07 & -0.07 & 0.48 & 1 & 1 \\
P-value & 0.88 & 0.88 & 0.28 & - & - \\
Weathering $(r)$ & $\mathbf{0 . 9 3}$ & $\mathbf{0 . 6 8}$ & 0.48 & 0.12 & \\
P-value & $\mathbf{0 . 0 0 3}$ & $\mathbf{0 . 0 9}$ & 0.27 & 0.8 & - \\
RMR $(r)$ & 0.44 & 0.34 & 0.15 & -0.12 & 0.27 \\
$P$-value & 0.32 & 0.46 & 0.75 & 0.8 & 0.56 \\
\hline
\end{tabular}

RMR - Rock Mass Rating. 
Peak acceleration and peak velocity have a strong positive correlation $(r>0.7)$ with a statistically significant result $(p<0.05)$. This validates the deformation time series charts, showing that continued velocity increases up to the point of failure and supports the accelerating creep theory.

Peak velocity has a strong positive and statistically significant $(r>0.7$ and $p<0.05)$ correlation to failure size, suggesting that as the failure size increases so does the peak velocity. A similar result was observed by Carla et al. (2017a) that suggested larger failures can accommodate higher total displacements and velocity rates due to multiple planes interacting and interlocking.

No significant correlation was observed in the current dataset between failure size and onset of tertiary creep. This could be due to the limited historical record in a number of failures where SSR monitoring was started shortly before collapse.

Weathering has a strong correlation to acceleration and to a lesser extent velocity. The results for weathering should be used with caution as the values are ordinal. However, a basic interpretation may suggest that as the degree of weathering increases (i.e. becoming more soil like) the total deformation, acceleration and velocity rates increase. This is broadly expected as soils are more likely to exhibit ductile behaviour which would result in higher strain rates (i.e. velocity).

Overall confidence in the statistical analysis may be reduced due to the small dataset $(n=7)$. In addition, it was assumed all the variables were independent, although may have a high interrelationship between variables. For example, the intensity of weathering will be related to a lower RMR value.

The statistical analysis concluded the variables with the strongest correlation were peak acceleration, peak velocity, failure size and weathering. Therefore, it is recommended practitioners recognise the expected failure mechanism, size and weathering condition of the slope when setting TARP levels.

Six log-velocity and log-acceleration plots were created to identify trends for the individual variables of; weathering, RMR, rock type, failure mode, duration of the final increment of displacement leading to failure (i.e. tertiary creep phase) and failure mass. Log-log plots were used to minimise the effect of the skewed result of Failure \#2, which has a high peak velocity rate.

Weathering is clustered by the degree of weathering with higher values resulting in higher peak accelerations and peak velocity rates (Figure 3(a)). This indicates that soil like material fails at higher total acceleration and velocity rates when compared to rock. Rock material is likely to be brittle and structurally controlled, therefore not able to accommodate large deformation rates (Carla et al. 2017a).

It was expected that RMR results would exhibit a similar trend to weathering with higher RMR material failing at lower acceleration and velocity rates indicating a stronger and more brittle rock mass. However, no clear trends were observed in the RMR plot (Figure $3(b)$ ). This may be due to the narrow range and low RMR values (range of 15 and maximum value 30 ) in the case examples that did not provide a sufficiently broad dataset to identify differences.

Weaker rock types such as Terre Rouge (soil) and contact zones failed at higher velocity and acceleration rates as opposed to the R3 rock (Figure 3(c)). This result supports a similar observation from weathering and highlights the interrelationship between variables that may exist.

The two different failure modes recorded (hybrid and rock mass) appear to be grouped separately (Figure 3(d)). A straight line was fitted between the peak velocity and peak acceleration values for rock mass failures indicating a power relationship may exist. Whereas, hybrid events fit a convex curve indicating increasing peak acceleration rates compared to peak velocity rates. The effect of a discrete weak plane as part of the failure mode may potentially enable rapid sliding to occur once the yield point is exceeded resulting in higher acceleration rates.

No clear trend was observed for the start of tertiary creep for the different events (Figure 3(e)). Possibly, the late deployment of the SSR system in a number of examples resulted in a small time series of data collected before collapse. As a result, only the tertiary creep phase was recorded which may provide erroneous results. 
Failure size is clustered, with larger events having higher peak velocity and acceleration rates (Figure 3(f)). This observation mirrors the statistical analysis presented above and supports the assumption that larger failures are able to accommodate higher total displacement rates.
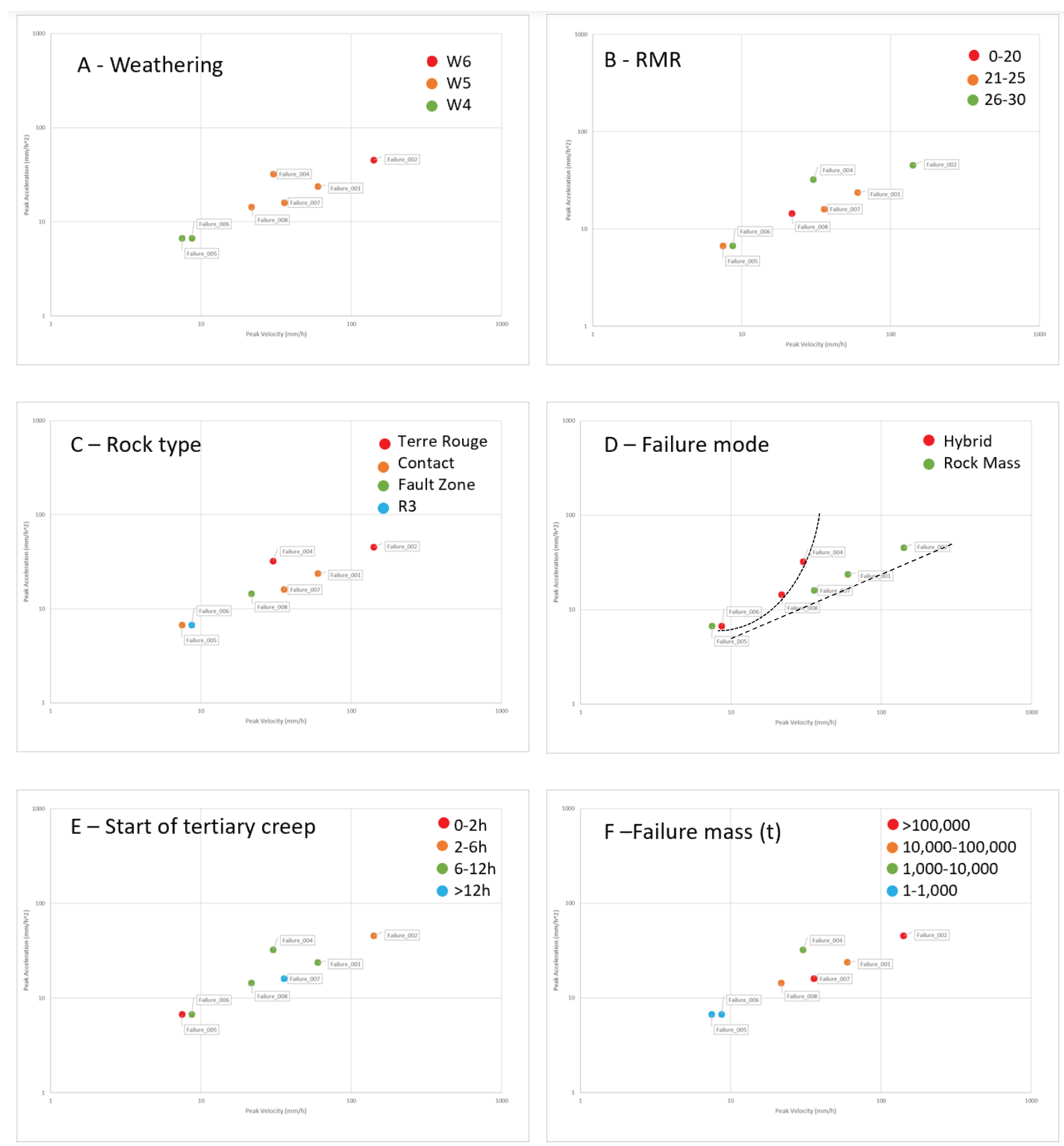

Figure 3 Analysis of log-velocity versus log-acceleration for study site examples for weathering, Rock Mass Rating, rock type, failure mode, start of tertiary creep and failure mass. Note: the trend lines indicated in Figure 3(d) are approximations

Based on the log plots and statistical analysis a TARP was developed for the study site with the aim of triggering alarms 12-24 hours prior to collapse (Figure 4). The TARP compares two variables, velocity and estimated failure size to ensure mitigating actions are implemented proportional to the risk posed by the potential failure. The two variables were selected as they have a positive correlation, visual analysis grouping and both can be practically implemented. Weathering was omitted from the TARP as the slopes were all moderately to completely weathered and therefore expected to behave similarly. However, as the pit 
progresses it is recommended that areas of the slopes which are less weathered have separate thresholds developed. Since the implementation of the TARP all failures successfully triggered alarms at an early stage and progressed through the risk matrix as the velocity rates increased. Appropriate and proportional actions were implemented, mitigating the risk to equipment and personnel. It is recommended that the TARP is continually reviewed after every failure event to verify alarms are triggering effectively.

\section{Deformation rate or velocity}

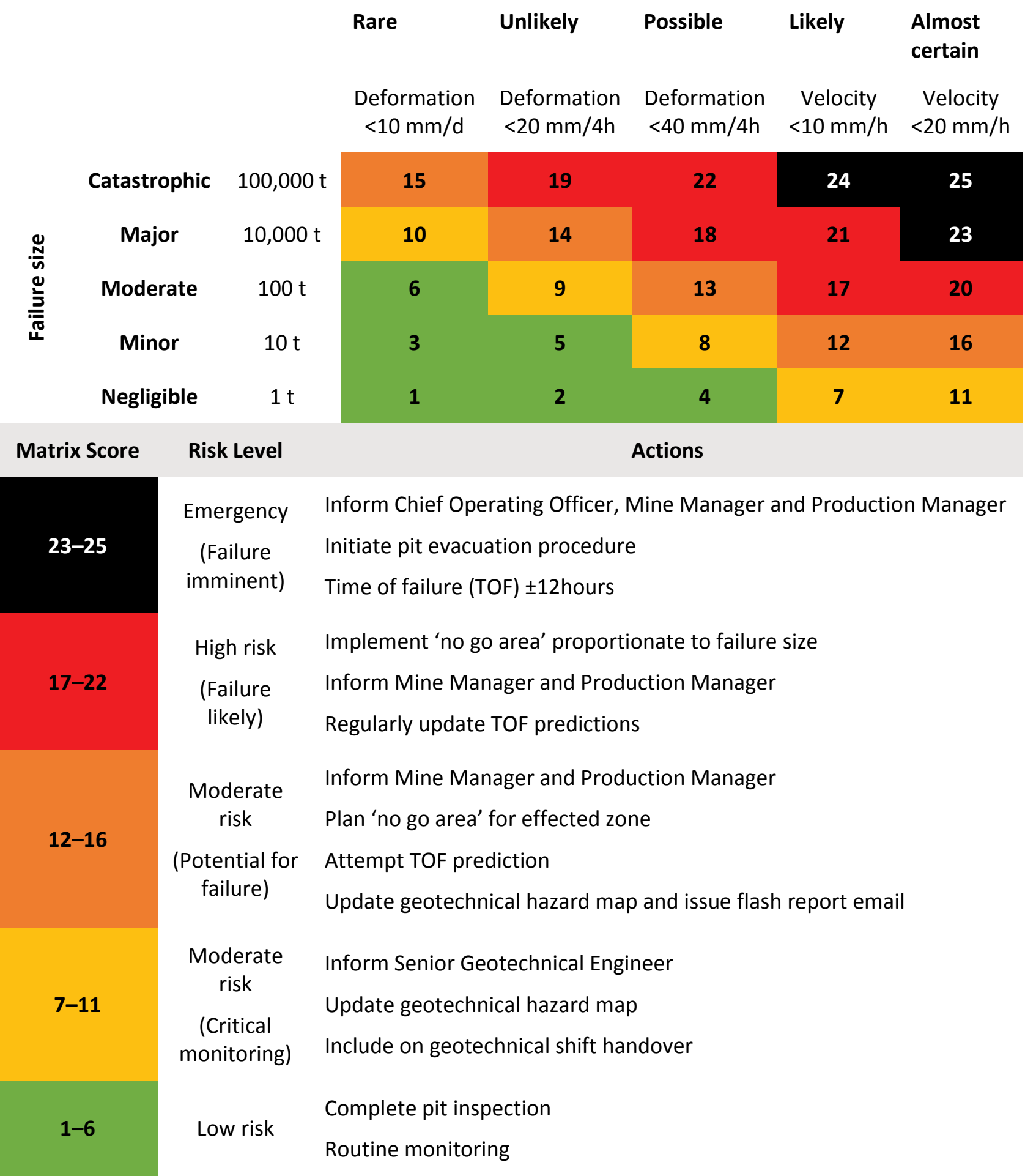

Figure 4 The slope deformation trigger action response plan implemented based on the failures that occurred at the study site 
The single variable analysis completed was adequate in providing observations between the seven study site examples. However, it was unable to identify the relative importance of each variable which would help in setting TARP thresholds based on observations of the slope at other sites (e.g. weathering, RMR or failure size). Further, the analysis completed assumed each variable was independent. However, it is likely a strong interrelationship exists between many of the variables. For example, a rock slope with a high RMR is likely to be less weathered. Therefore, it is recommended future analysis accounts for interrelationship.

\subsection{Comparison to other failures}

To understand inter-site failure trends the study site examples were compared to other published failure events from open pit mining examples (Carla et al. 2017a) and landslide events (Mazzanti et al. 2015) monitored by terrestrial SSR. Figure 5 outlines 19 events plotted by peak velocity and peak acceleration. The size of the circles represents the failure tonnage and colours indicating failure mode.

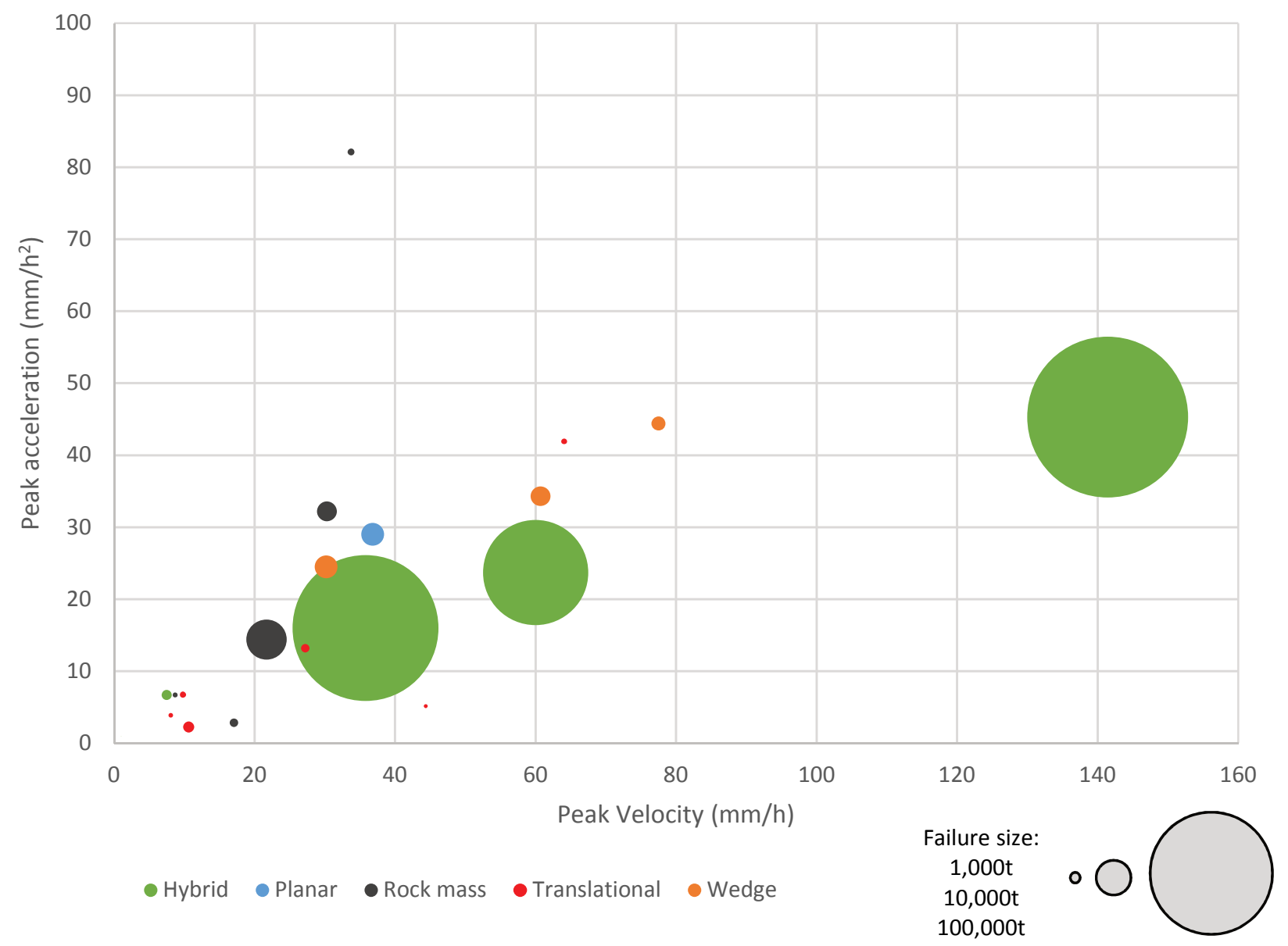

Figure 5 Comparison of peak velocity, acceleration rates, failure size and mode for case study examples and additional published sources (Mazzanti et al. 2015; Carla et al. 2017a)

Small failures $(<1,000 \mathrm{t})$ have the highest clustering in the bottom left hand corner of the figure occurring with a peak velocity less than $20 \mathrm{~mm} / \mathrm{h}$ and peak acceleration less than $15 \mathrm{~mm} / \mathrm{h}^{2}$. However, four events occurred outside this range with one extreme anomalous event failing with a velocity of $33 \mathrm{~mm} / \mathrm{h}$ and acceleration of $82 \mathrm{~mm} / \mathrm{h}^{2}$. This event was taken from a study by Mazzanti et al. (2015) of 10 shallow landslide events mostly triggered by rainfall. It is possible the anomalous value was a result of heavy rain prior to the event.

Planar and wedge type failures had a peak velocity rate between $30 \mathrm{~mm} / \mathrm{h}$ to $80 \mathrm{~mm} / \mathrm{h}$ and peak acceleration between $25 \mathrm{~mm} / \mathrm{h}^{2}$ to $45 \mathrm{~mm} / \mathrm{h}^{2}$. The three large hybrid events appear to overlap the range of velocity and 
acceleration rate observed for the planar and wedge failure modes. Notably the hybrid events are on average 50 times larger in mass (average tonnage for hybrid 150,000 t versus average tonnage planar and wedge $3,000 \mathrm{t}$ ). However, these events have broadly similar velocity and acceleration rates. Carla et al. (2017a) suggested that larger failures can accommodate higher total displacements and velocity rates due to multiple planes interacting and interlocking in larger events and the results presented here would support that statement up to approximately 3,000 t. Failure events greater than 3,000 t do not appear to have clear grouping by failure size, thus other factors associated with failures are likely to be controlling the peak acceleration and velocity rates.

In general, grouping of results was observed as a combination of failure mode and failure size (less than $1,000 \mathrm{t}$ and greater than 3,000 t). The visual grouping observed in this study supports the suggestion by Newcomen \& Dick (2016) that sorting data by failure mode would potentially identify subsets which exist within the examples. It is recommended that additional variables (e.g. RMR, GSI, weathering and lithology) are included in later studies to provide further insights into factors that affect the peak velocity and acceleration rates.

\section{$5 \quad$ Conclusion and recommendations}

The inverse velocity method has been proven on multiple occasions to predict TOF. Appropriate assessment of SSR scan times, LOS sensitive and VCP can aid in improving predictions. However, the current challenge facing practitioners is setting SSR alarm thresholds that trigger 12-24 hour prior to failure to enable forecasting. This study has utilised the methods suggested by Carla et al. (2017a) to identify broader signatures that can be used in TARP plans using velocity-acceleration plots.

The results from the study site examples show that peak acceleration and peak velocity rates of failures are grouped by multiple characteristics. Specifically, the strongest grouping was observed by the degree of weathering and failure sizes for the different events. The study site TARP is presented that combines actions based on velocity and failure size. To date the TARP has worked effectively with all slope instability events identified at an early stage with mitigating actions implemented to provide minimum practical time required for safe evacuation. It is recommended that every time a failure event occurs the analysis is updated and TARP thresholds revised.

The dataset of 19 failure events showed grouping as a combination of failure size and failure mode up to approximately 3,000 t. Failure events greater than 3,000 t do not appear to have clear grouping by failure size or failure mode suggesting other factors are controlling the peak acceleration and velocity rates. Therefore, the results from this study suggest the TARP should first consider different trigger thresholds based on the expected failure mode. Following this trigger levels can be refined considering failure size estimated on either small $(<1,000 \mathrm{t})$ or large $(>3,000 \mathrm{t})$ events.

The main limitation of the present study was the small dataset available from the study site and other published failures and variables for each event. This suggests the need for a larger database incorporating failures monitored using SSR data. Farina et al. (2018b) also made a similar recommendation and provided a workflow for the collection of data so a more reliable analysis method could be developed.

More broadly, future research is required to keep pace with the increased adoption of automated monitoring systems collecting larger quantities of temporal data. For example, a comparison of the failure signatures identified from multiple monitoring systems (e.g. SSR, robotic total station, piezometer and InSAR) for different failure events may provide larger, more robust databases for further analysis (Carla et al. 2018; Farina et al. 2018a) in order to inform TARP and provide safe operational environments.

\section{Acknowledgement}

The authors thank the mining company for the use of data in the report. 


\section{References}

Arosio, D \& Harries, N 2010, 'Predicting slope collapse using slope stability radar deformation data', International Society for Rock Mechanics and Rock Engineering.

Bar, N, Parker, R \& Thomas, S 2016, 'Managing landslide risks associated with erosion-driven instabilities using near real-time deformation monitoring systems', Rock Mechanics and Rock Engineering: From the Past to the Future.

Cabrejo-Lievano, AG 2013, 'Analysis of failures in open pit mines and considerations of the uncertainty when predicting collapses', in PM Dight (ed.), Proceedings of the 2013 International Symposium on Slope Stability in Open Pit Mining and Civil Engineering, Australian Centre for Geomechanics, Perth, pp. 483-497.

Carla, T, Farina, P, Intrieri, E, Botsialas, K \& Casagli, N 2017a, 'On the monitoring and early warning of brittle slope failures in hard rock masses: Examples from an open-pit mine', Engineering Geology, vol. 228, pp. 71-81, https://doi.org/10.1016/ j.enggeo.2017.08.007

Carla, T, Intrieri, E, Di Traglia, F, Nolesini, T, Gigli, G \& Casagli, N 2017b, 'Guidelines on the use of inverse velocity method as a tool for setting alarm thresholds and forecasting landslides and structure collapses', Landslides, vol. 14, no. 2, pp. 517-534, https://doi.org/10.1007/s10346-016-0731-5

Carla, T, Farina, P, Intrieri, E, Ketizmen, H \& Casagli, N 2018, 'Integration of ground-based radar and satellite InSAR data for the analysis of an unexpected slope failure in an open-pit mine', Engineering Geology, vol. 235, pp. 39-52, https://doi.org/10.1016/j.enggeo.2018.01.021

Crosta, GB, Agliardi, F, Rivolta, C, Alberti, S \& Dei Cas, L 2017, 'Long-term evolution and early warning strategies for complex rockslides by real-time monitoring', Landslides, vol. 14, no. 5, pp. 1615-1632, https://doi.org/10.1007/s10346-017-0817-8

Dick, GJ, Eberhardt, E, Cabrejo-Liévano, AG, Stead, D \& Rose, ND 2015, 'Development of an early-warning time-of-failure analysis methodology for open-pit mine slopes utilizing ground-based slope stability radar monitoring data', Canadian Geotechnical Journal, vol. 52, no. 4, pp. 515-529, https://doi.org/10.1139/cgj-2014-0028

Farina, P, Carla, T, Intrieri, E, Ketizmen, H \& Casagli, N 2018a, 'Characterisation of a large slope failure in an open-pit mine through the back-analysis of satellite InSAR and ground-based radar data', Proceedings of the 2018 International Symposium on Slope Stability in Open Pit Mining and Civil Engineering, BCO Congresos, Barcelona.

Farina, P, Carla, T, Intrieri, E \& Casagli, N 2018b, 'Identifying 'signatures' of slope failure conditions in open pit mines to support the set-up of alarms: a possible workflow', Proceedings of the 2018 International Symposium on Slope Stability in Open Pit Mining and Civil Engineering, Seville, Spain.

Federico, A, Popescu, M, Elia, G, Fidelibus, C, Internò, G \& Murianni, A 2012, 'Prediction of time to slope failure: a general framework', Environmental Earth Sciences, vol. 66, no. 1, pp. 245-256, https://doi.org/10.1007/s12665-011-1231-5

Fukuzono, T 1985, 'A new method for predicting the failure time of a slope', Proceedings of 4th International Conference and Field Workshop on Landslides, pp. 145-150.

Harries, N, Noon, D \& Rowley, K 2006, 'Case studies of slope stability radar used in open cut mines', International Symposium on Stability of Rock Slopes, pp. 335-342.

Harries, N, Noon, D, Pritchett, H \& Bates, D 2009, 'Slope stability radar for managing rock fall risks in open cut mines', Rock Engineering in Difficult Conditions, Proceedings of the 3rd Canada-US Rock Mechanics Symposium, pp. 9-15.

Maton, T 2002, Geotechnical Management at the Martha Pit, unpublished.

Mazzanti, P, Bozzano, F, Cipriani, I \& Prestininzi, A 2015, 'New insights into the temporal prediction of landslides by a terrestrial SAR interferometry monitoring case study', Landslides, vol. 12, no. 1, pp. 55-68, https://doi.org/10.1007/s10346-014-0469-x

Mufundirwa, A, Fujii, Y \& Kodama, J 2010, 'A new practical method for prediction of geomechanical failure-time', International Journal of Rock Mechanics and Mining Sciences, vol. 47, no. 7, pp. 1079-1090, https://doi.org/10.1016/j.ijrmms.2010.07.001

Newcomen, W \& Dick, G 2016, 'An update to the strain-based approach to pit wall failure prediction, and a justification for slope monitoring', Journal of the Southern African Institute of Mining and Metallurgy, vol. 116, no. 5, pp. 379-385, http://dx.doi.org/10.17159/2411-9717/2016/v116n5a3

Osasan, KS \& Afeni, TB 2010, 'Review of surface mine slope monitoring techniques', Journal of Mining Science, vol. 46, no. 2, pp. 177-186, https://doi.org/10.1007/s10913-010-0023-8

Read, J \& Stacey, P 2009, Guidelines for Open Pit Slope Design, CSIRO Publishing, Australia.

Rose, ND \& Hungr, O 2007, 'Forecasting potential rock slope failure in open pit mines using the inverse-velocity method', International Journal of Rock Mechanics and Mining Sciences, vol. 44, no. 2, pp. 308-320, https://doi.org/10.1016/j.ijrmms.2006.07.014

Saito, M 1965, 'Forecasting the time of occurrence of a slope failure', Proceeding of the 6th International Conference of Soil Mechanics and Foundation Engineering, pp. 537-541.

Vaziri, A, Moore, L \& Ali, H 2010, 'Monitoring systems for warning impending failures in slopes and open pit mines', Natural Hazards, vol. 55, no. 2, pp. 501-512, https://doi.org/10.1007/s11069-010-9542-5

Venter, J, Kuzmanovic, A \& Wessels, SDN 2013, 'An evaluation of the CUSUM and inverse velocity methods of failure prediction based on two open pit instabilities in the Pilbara', Proceedings of the 2013 International Symposium on Slope Stability in Open Pit Mining and Civil Engineering, Australian Centre for Geomechanics, Perth, pp. 1061-1076. 